\title{
Redundant Coordinates in Quantum Mechanics
}

\author{
Eric Toombs \\ Tucker Carrington Jr. \\ Queen's University, Kingston, Ontario KrL 3N6, Canada
}

\begin{abstract}
We present a kinetic energy operator and inner product that can be used to solve the Schroedinger equation in redundant coordinates. The goal is to develop equations and a computational procedure that can be used with $N$ coordinates for a system with $M$ degrees of freedom, where $N>M$. In chemical physics, this might be useful for exploiting symmetry or exploiting certain representations of potentials. Calculations demonstrate that the ideas work.
\end{abstract}




\section{INTRODUCTION}

In this paper we develop a theory that enables one to solve the Schroedinger equation in redundant coordinates. This is of interest in and of itself, but should also have practical applications. In molecular physics, using redundant coordinates would facilitate exploiting symmetry. Take $\mathrm{NH}_{3}$, for example. If it were possible to write and solve the ro-vibrational Schroedinger equation in a set of coordinates that includes $3 \mathrm{NH}$ stretches, $3 \mathrm{HNH}$ angles and one out of plane angle, it would be easier to take advantage of the permutation symmetry of the three identical hydrogen nuclei. Using redundant coordinates will also make compact representations of potential energy surfaces (PES) in inter-atomic coordinates more useful. Potential energy surfaces can often be represented as a sum of terms depending on subsets of inter-atomic distances, [1]

$$
V(\boldsymbol{y})=\sum_{i} V_{i}\left(y_{i}\right)+\sum_{i j} V_{i j}\left(y_{i}, y_{j}\right)+\ldots
$$

One way to fully exploit the compactness of this representation is to solve the vibrational Schroedinger equation in inter-atomic distances $\boldsymbol{y}$ with a product basis. When terms with at most four coordinates are required then potential matrix elements can be computed by doing at most four dimensional quadrature. However, for molecules for which the number of atoms, $N_{a}$, is 5 or more, the number of inter-atomic distances is greater than the number of vibrational degrees of freedom, $3 N_{a}-6$; the coordinates are redundant and one does not know how to make a matrix representation of the Schroedinger equation and therefore cannot profit from the compactness of Eq. (1). It is also straightforward to exploit symmetry in inter-atomic coordinates. Because it is not known how to solve the Schroedinger equation in redundant coordinates, it is common use a kinetic energy operator (KEO) in non-redundant coordinates $\boldsymbol{x}$, the transformation from $\boldsymbol{x}$ to $\boldsymbol{y}$, and quadrature or collocation. [3-5] In general, $3 N_{a}-6$ dimensional quadrature is required. For high-dimensional problems many points are required. [6]

Using the theory of this paper, it is possible to solve the Schroedinger equation in redundant coordinates and obtain exact results, i.e. the same results one obtains by solving the Schroedinger equation in non-redundant coordinates. Two issues require some thought: 1) what is the kinetic energy operator (KEO) in redundant coordinates; 2) how does one calculate inner products of functions of redundant coordinates. 
The redundant coordinates satisfy a constraint equation. There is a lot of work on formulating the Schroedinger equation with constraints. [7-9] However, in that work one wishes to solve the Schroedinger equation using fewer coordinates and in this paper we are using more coordinates. We require constraints not because we are reducing the size of configuration space, but because we expand configuration space. Other authors impose constraints [10-12] that modify the physical properties of the system by confining it to a lower-dimensional region of the original configuration space. In this paper, it is by imposing constraints that the physical properties of the system are not altered.

We develop ideas that can be used to solve the Schroedinger equation for a system with $M$ degrees of freedom using $N>M$ coordinates. It could be solved with $M$ regular coordinates which we denote $\boldsymbol{x}$. We use, instead, $N$ redundant coordinates which we denote $\boldsymbol{y}$. Values of $\boldsymbol{y}$ that are physical are constrained to an $M$-dimensional subspace of the full $N$-dimensional coordinate space that we call the shell. A simple example is the motion of a particle on a sphere of unit radius. An obvious choice of the regular coordinates is the polar angles $\theta$ and $\phi$. We want to be able to solve the Schroedinger equation using three Cartesian coordinates, $n_{x}, n_{y}, n_{z}$ that satisfy the constraint $n_{x}^{2}+n_{y}^{2}+n_{z}^{2}=1$.

\section{STRATEGY AND NOTATION}

One way to proceed is: 1) add $N-M$ new coordinates $\boldsymbol{k}$ to $\boldsymbol{x}$ to obtain a new set of coordinates, denoted $\boldsymbol{z} .2$ ) choose an inner product and a KEO that can be used with the $\boldsymbol{z}$ coordinates so that energies computed with the $\boldsymbol{z}$ coordinates are equal to those computed with the $\boldsymbol{x}$ coordinates; 3 ) start with the inner product and the KEO in the $\boldsymbol{z}$ coordinates and transform to obtain an inner product and a KEO in $\boldsymbol{y}$ coordinates. We call $\boldsymbol{z}$ the augmented coordinates. On the shell $\boldsymbol{k}=\boldsymbol{k}_{\mathbf{0}} ; \boldsymbol{k}_{\mathbf{0}}$ is determined by the physical problem we are solving. We define $\boldsymbol{Y}_{\mathbf{0}}(\boldsymbol{x})$ to be the function that maps values of $\boldsymbol{x}$ to values of $\boldsymbol{y}$ in the physical region, i.e. on shell. We define $\boldsymbol{Y}(\boldsymbol{z})$ to be the function that maps values of $\boldsymbol{z}$ to values of $\boldsymbol{y}$ in the extended space i.e. on shell and off shell. It is imperative that $\boldsymbol{y}=\boldsymbol{Y}\left(\boldsymbol{x}, \boldsymbol{k}_{\mathbf{0}}\right)=\boldsymbol{Y}_{\mathbf{0}}(\boldsymbol{x})$ when $\boldsymbol{y}$ is on-shell. For the particle on a sphere example of the introduction, $\boldsymbol{x}=\{\theta, \phi\}, k=n_{x}^{2}+n_{y}^{2}+n_{z}^{2}, k_{0}=1$ and $\boldsymbol{y}=\left\{n_{x}, n_{y}, n_{z}\right\}$ on-shell. With this notation, $\psi_{y}\left(\boldsymbol{Y}\left(\boldsymbol{x}, \boldsymbol{k}_{\mathbf{0}}\right)\right)=\psi_{x}(x)$. The subscript indicates the coordinates on which a 
wavefunction depends. In this paper, all functions of $\boldsymbol{z}$ satisfy,

$$
\psi_{z}(\boldsymbol{z})=\psi_{z}(\boldsymbol{x}, \boldsymbol{k})=\psi_{x}(\boldsymbol{x})
$$

$\boldsymbol{Y}(\boldsymbol{z})$ has an inverse $\boldsymbol{Y}^{-\mathbf{1}}(\boldsymbol{y})=\boldsymbol{z}$. The $k$ component(s) of $\boldsymbol{Y}^{-\mathbf{1}}(\boldsymbol{y})=\boldsymbol{z}$ is (are) $\left(\boldsymbol{Y}^{-1}\right)_{\boldsymbol{k}}(\boldsymbol{y})=\boldsymbol{k}$ and on-shell $\left(\boldsymbol{Y}^{-1}\right)_{\boldsymbol{k}}(\boldsymbol{y})=\boldsymbol{k}_{\mathbf{0}}$. There is one component of $\boldsymbol{Y}^{-\mathbf{1}}(\boldsymbol{y})$ for each of the $\boldsymbol{z}$ coordinates.

\section{CALCULATING INNER PRODUCTS IN REDUNDANT COORDINATES}

To do calculations in $\boldsymbol{y}$ it is imperative that we have a $\boldsymbol{y}$ inner product, which we will need to calculate matrix elements. In the regular coordinates $\boldsymbol{x}$, the inner product of two functions is (we ignore spin in this paper),

$$
\left\langle\psi_{x}, \phi_{x}\right\rangle_{x}=\int \rho_{x}(\boldsymbol{x}) \psi_{x}(\boldsymbol{x})^{*} \phi_{x}(\boldsymbol{x}) d \boldsymbol{x}
$$

where $\rho_{x} d \boldsymbol{x}$ is the volume element. To solve the Schroedinger equation in the $\boldsymbol{y}$ coordinates we need to define an inner product $\left\langle\psi_{y}, \phi_{y}\right\rangle_{y}$ so that

$$
\left\langle\psi_{y}, \phi_{y}\right\rangle_{y}=\left\langle\psi_{x}, \phi_{x}\right\rangle_{x}
$$

If $N$ and $M$ were equal, one would obtain the $\boldsymbol{y}$ volume element from the $\boldsymbol{x}$ volume element by multiplying by a Jacobian. That is not possible when $N>M$ because the matrix of derivatives is not square. Instead, we first obtain a useful volume element in $\boldsymbol{z}$ coordinates and from it derive a volume element in $\boldsymbol{y}$ coordinates. The volume element in $\boldsymbol{z}$ coordinates is obtained from

$$
\begin{gathered}
\left\langle\psi_{z}, \phi_{z}\right\rangle_{z}=\int \rho_{z}(\boldsymbol{z}) \psi_{z}(\boldsymbol{z})^{*} \phi_{z}(\boldsymbol{z}) \delta\left(\boldsymbol{k}-\boldsymbol{k}_{\mathbf{0}}\right) d \boldsymbol{z} \\
=\left\langle\psi_{x}, \phi_{x}\right\rangle_{x}
\end{gathered}
$$

where

$$
\rho_{z}(\boldsymbol{z})=\rho_{z}(\boldsymbol{x}, \boldsymbol{k})=\rho_{x}(\boldsymbol{x}) .
$$

The Dirac delta filters the integration domain to just the physically possible values of $\boldsymbol{z}$. In practice we use a window function rather than a Dirac delta function. Transforming to $\boldsymbol{y}$ coordinates is easy from here, because both $\boldsymbol{y}$ and $\boldsymbol{z}$ have $N$ coordinates. After doing so, 
the delta filter remains.

$$
\begin{aligned}
\left\langle\psi_{y}, \phi_{y}\right\rangle_{y} & =\int_{y} \rho_{y}(\boldsymbol{y}) \psi_{y}(\boldsymbol{y})^{*} \phi_{y}(\boldsymbol{y}) \delta\left(\left(\boldsymbol{Y}^{-\mathbf{1}}\right)_{k}(\boldsymbol{y})-\boldsymbol{k}_{\mathbf{0}}\right) d \boldsymbol{y} \\
& =\left\langle\psi_{x}, \phi_{x}\right\rangle_{x}
\end{aligned}
$$

where

$$
\begin{gathered}
\phi_{y}(\boldsymbol{y})=\phi_{z}\left(\boldsymbol{Y}^{-\mathbf{1}}(\boldsymbol{y})\right) \\
\rho_{y}(\boldsymbol{y})=\frac{\rho_{x}\left(\left(\boldsymbol{Y}^{-\mathbf{1}}\right)_{\boldsymbol{x}}(\boldsymbol{y})\right)}{\operatorname{det}(\Lambda(\boldsymbol{y}))}
\end{gathered}
$$

and

$$
\Lambda(\boldsymbol{y})=\hat{\Lambda}\left(\boldsymbol{Y}^{-\mathbf{1}}(\boldsymbol{y})\right) ; \quad \text { with }(\hat{\Lambda}(\boldsymbol{z}))_{i j}=\frac{\partial Y_{i}}{\partial z_{j}}(\boldsymbol{z}) .
$$

$\frac{1}{\operatorname{det}(\Lambda(\boldsymbol{y}))}$ is the Jacobian for the transformation from $\boldsymbol{z}$ to $\boldsymbol{y}$, expressed as a function of $\boldsymbol{y}$. Using Eq. (8), it will be possible to compute matrix elements of the Hamiltonian in $\boldsymbol{y}$.

$\left\langle\psi_{y}, \phi_{y}\right\rangle_{y}$ is defined so that Eq. (4) is satisfied. It is also satisfied if $\rho_{z}$ of Eq. (5), from which $\rho_{y}$ is obtained, is multiplied by a function $S(\boldsymbol{k})$ for which $S\left(\boldsymbol{k}_{\mathbf{0}}\right)=1$. We find that it is possible to choose $S(\boldsymbol{k})$ to simplify calculating matrix elements of the KEO. The most general form of $\rho_{y}(\boldsymbol{y})$ is therefore,

$$
\rho_{y}(\boldsymbol{y})=S(\boldsymbol{k}) \frac{\rho_{x}\left(\left(\boldsymbol{Y}^{-\mathbf{1}}\right)_{\boldsymbol{x}}(\boldsymbol{y})\right)}{\operatorname{det}(\Lambda(\boldsymbol{y}))} .
$$

\section{A KINETIC ENERGY OPERATOR IN REDUNDANT COORDINATES}

The kinetic energy in the regular coordinates can be written as (the Einstein summation convention and atomic units are used in this paper)

$$
T_{x}=-\frac{1}{2 \rho_{x}} \frac{\partial}{\partial x^{a}}\left(\rho_{x} \tau_{x}^{a b} \frac{\partial}{\partial x^{b}}\right) .
$$

This is a Podolsky- or Laplace-Beltrami-type operator, $[13,14]$ where $\rho_{x} d \boldsymbol{x}$ is the volume element. The KEO in the $\boldsymbol{y}$ coordinates has the same form,

$$
T_{y}=-\frac{1}{2 \rho_{y}} \frac{\partial}{\partial y^{a}}\left(\rho_{y} \tau_{y}^{a b} \frac{\partial}{\partial y^{b}}\right)
$$

From Eq. (14), it is not simple to derive the KEO in the redundant $\boldsymbol{y}$ coordinates. A simple application of the chain rule does not work because the correct KEO must take the constraints on the redundant coordinates into account. In addition, the matrix $\boldsymbol{\tau}_{\boldsymbol{y}}$, obtained 
from the chain rule, will be singular. Therefore, it is not possible to obtain $\rho_{y}$ in the standard fashion, from the inverse of the square root of the determinant of $\boldsymbol{\tau}_{\boldsymbol{y}}$. On the other hand, transforming Eq. (14) to augmented coordinates is straightforward. This KEO is

$$
T_{z}=-\frac{1}{2 \rho_{z}} \frac{\partial}{\partial z^{a}}\left(\rho_{z} \tau_{z}^{a b} \frac{\partial}{\partial z^{b}}\right)
$$

where $\boldsymbol{\tau}_{\boldsymbol{z}}$ is obtained from $\boldsymbol{\tau}_{\boldsymbol{x}}$ by extending it with zeros,

$$
\tau_{z}=\left[\begin{array}{cc}
\tau_{x} & 0 \\
0 & 0
\end{array}\right] .
$$

$\rho_{z}=\rho_{x}$, for any value of $\boldsymbol{k}$. According to the usual transformation rules

$$
\tau_{y}(y)=\Lambda(y)\left[\begin{array}{cc}
\tau_{x}\left(\left(Y^{-1}\right)_{x}(y)\right) & 0 \\
0 & 0
\end{array}\right] \Lambda^{T}(y),
$$

where $\boldsymbol{\Lambda}$ is defined as in equation Eq. (12). However, it is not possible to find $\rho_{y}$ in the standard fashion because $\boldsymbol{\tau}_{\boldsymbol{y}}$ is singular. Instead, the volume element is given by equation 11 .

The most straightforward way to derive the KEO in the $\boldsymbol{y}$ coordinates requires computing $\tau_{y}$ from Eq. (18). This requires multiplying matrices because $\tau_{y}=\Lambda \tau_{z} \Lambda^{T}$. When $\boldsymbol{x}$ or $\boldsymbol{y}$ is composed of many smaller vectors - possibly an unspecified number, i.e. $\boldsymbol{y}=\left(\boldsymbol{R}, \boldsymbol{r}_{1}, \boldsymbol{r}_{2}, \ldots\right)$ - the matrix multiplication is cumbersome. We begin with the classical kinetic energy written in terms of $\boldsymbol{p}_{\boldsymbol{z}}$ and then derive equations for the components of $\boldsymbol{p}_{\boldsymbol{z}}$ in terms of the components of $\boldsymbol{p}_{\boldsymbol{y}}$. This procedure is illustrated in section VI for a particular example. We begin with

$$
T=\frac{1}{2} \boldsymbol{p}_{\boldsymbol{z}}^{\boldsymbol{T}} \boldsymbol{\tau}_{\boldsymbol{z}} \boldsymbol{p}_{\boldsymbol{z}}
$$

We know that

$$
\begin{gathered}
\dot{y}=\Lambda \dot{z} \\
p_{z}=\Lambda^{T} p_{y} .
\end{gathered}
$$

From these equations, it is easy to see that

$$
p_{z}^{T} \dot{z}=p_{y}^{T} \dot{y}
$$

Using Eq. (22), it is now straightforward to obtain an equation for a single component of $\boldsymbol{p}_{\boldsymbol{z}}$ in terms of $\boldsymbol{p}_{\boldsymbol{y}}$ by zeroing all but the corresponding component of $\dot{\boldsymbol{z}}$. Equations for the components can then be substituted into Eq. (19) to get $\boldsymbol{\tau}_{\boldsymbol{y}}$. 


\section{EXAMPLE: A 4D KEO FOR A PARTICLE MOVING IN 3D}

The first - and simplest - system that will be used as an example is the 3D motion of a single particle. We start with the three Cartesian coordinates of the particle, $\boldsymbol{x} \in \mathbb{R}^{3}$, define a set of four redundant $\boldsymbol{y}$ coordinates and then find the KEO in the $\boldsymbol{y}$ coordinates. The $\boldsymbol{y}$ coordinates are: $r=|\boldsymbol{x}|$, the particle's distance from the origin, and the three Cartesian components of a unit vector $\boldsymbol{n}$ pointing from the origin to the particle. On the shell, the three components are,

$$
n_{x}=\frac{x_{x}}{|\boldsymbol{x}|} ; n_{y}=\frac{x_{y}}{|\boldsymbol{x}|} ; n_{z}=\frac{x_{z}}{|\boldsymbol{x}|} .
$$

Note that $\boldsymbol{n}$ is not a unit vector off-shell. On-shell and off-shell, $\boldsymbol{n}$ 's norm is totally unrelated to $r$. The coordinate transformation we must make is

$$
\boldsymbol{y}=(\boldsymbol{n}, \boldsymbol{r})=\boldsymbol{Y}(\boldsymbol{z})
$$

On-shell the redundant coordinates satisfy the constraint $|\boldsymbol{n}|=1$. As explained in section II, we need to transform from $\boldsymbol{x}$ to augmented coordinates and then to $\boldsymbol{y}$. To make the augmented coordinates $z=(\boldsymbol{x}, k)$, we add one coordinate to the set $\boldsymbol{x}$. The new coordinate is $k=|\boldsymbol{n}|$ and on-shell $k=k_{0}=1$. The function $\boldsymbol{Y}$ we use is

$$
\boldsymbol{y}=(\boldsymbol{n}, r)=\boldsymbol{Y}(\boldsymbol{x}, k)=\left(k \frac{\boldsymbol{x}}{|\boldsymbol{x}|},|\boldsymbol{x}|\right) .
$$

We start with

$$
T_{x}=-\frac{1}{2 m} \nabla_{x}^{T} \nabla_{x} .
$$

and $\rho_{x}=1$. We use $\boldsymbol{\tau}_{\boldsymbol{x}}=\mathbb{1}$ and just multiply by $\frac{1}{m}$ after deriving the KEO in the $\boldsymbol{y}$ coordinates. The $\boldsymbol{\tau}_{\boldsymbol{z}}$ matrix is constructed just as in equation 17 :

$$
\tau_{z}=\left[\begin{array}{ll}
\mathbb{1} & 0 \\
0 & 0
\end{array}\right] .
$$

Using Eq. (13) and $\rho_{x}=1$, we find

$$
\rho_{z}=S(k),
$$

where $S(k)$ can be any function for which $S\left(k_{0}\right)=1$. A good choice for it will be apparent after the transformation from $\rho_{z}$ to $\rho_{y}$.

To use Eq. (18) to obtain the KEO in $\boldsymbol{y}$ coordinates, we need $\hat{\Lambda}$. From equation Eq. (12),

$$
\hat{\Lambda}(\boldsymbol{z})=\left[\begin{array}{cc}
\frac{k}{|\boldsymbol{x}|}\left(\mathbb{1}-\frac{\boldsymbol{x} \boldsymbol{x}^{T}}{|\boldsymbol{x}|^{2}}\right) & \frac{\boldsymbol{x}}{|\boldsymbol{x}|} \\
\frac{\boldsymbol{x}^{T}}{|\boldsymbol{x}|} & 0
\end{array}\right]
$$


To get $\Lambda$ as a function of $\boldsymbol{y}$, we replace $\boldsymbol{z}$ in the previous equation with $\boldsymbol{Y}^{-1}(\boldsymbol{y})=\boldsymbol{z}$. It is easily proven that

$$
\boldsymbol{Y}^{-1}(\boldsymbol{n}, r)=(\boldsymbol{x}, k)=\left(r \frac{\boldsymbol{n}}{|\boldsymbol{n}|},|\boldsymbol{n}|\right) .
$$

Substituting this into the above, we get $\Lambda$ :

$$
\begin{aligned}
& \Lambda=\left[\begin{array}{cc}
\frac{|\boldsymbol{n}|}{r}\left(\mathbb{1}-\frac{\boldsymbol{n} \boldsymbol{n}^{T}}{|\boldsymbol{n}|^{2}}\right) & \frac{\boldsymbol{n}}{|\boldsymbol{n}|} \\
\frac{\boldsymbol{n}^{T}}{|\boldsymbol{n}|} & 0
\end{array}\right] \\
& \Lambda=\left[\begin{array}{cc}
\frac{|\boldsymbol{n}|}{r} P_{\perp} & \frac{\boldsymbol{n}}{|\boldsymbol{n}|} \\
\frac{\boldsymbol{n}^{T}}{|\boldsymbol{n}|} & 0
\end{array}\right] \text {, where } P_{\perp}=\mathbb{1}-\frac{\boldsymbol{n} \boldsymbol{n}^{T}}{|\boldsymbol{n}|^{2}}
\end{aligned}
$$

Note that $P_{\perp}$ is a projector onto the space orthogonal to $\boldsymbol{n}$, so $P_{\perp}^{2}=P_{\perp}$ and $P_{\perp} \boldsymbol{n}=0$. Therefore, from Eq. (18),

$$
\begin{aligned}
\boldsymbol{\tau}_{\boldsymbol{y}}(\boldsymbol{y}) & =\Lambda(\boldsymbol{y}) \boldsymbol{\tau}_{\boldsymbol{z}}\left(\boldsymbol{Y}^{-1}(\boldsymbol{y})\right) \Lambda^{T}(\boldsymbol{y}) \\
& =\left[\begin{array}{cc}
\frac{|\boldsymbol{n}|}{r} P_{\perp} & \frac{n}{|\boldsymbol{n}|} \\
\frac{\boldsymbol{n}^{T}}{|\boldsymbol{n}|} & 0
\end{array}\right]\left[\begin{array}{ll}
\mathbb{1} & 0 \\
0 & 0
\end{array}\right]\left[\begin{array}{cc}
\frac{|\boldsymbol{n}|}{r} P_{\perp} & \frac{n}{|\boldsymbol{n}|} \\
\frac{\boldsymbol{n}^{T}}{|\boldsymbol{n}|} & 0
\end{array}\right] \\
& =\left[\begin{array}{rr}
\frac{|\boldsymbol{n}|^{2}}{r^{2}} P_{\perp} \\
& 1
\end{array}\right] \\
\boldsymbol{\tau}_{\boldsymbol{y}} & =\left[\begin{array}{ll}
\frac{1}{r^{2}}\left(|\boldsymbol{n}|^{2}-\boldsymbol{n} \boldsymbol{n}^{T}\right) \\
\end{array}\right]
\end{aligned}
$$

In this paper, matrix elements that are not specified are zero.

We must also calculate the volume element. According Eq. (13):

$$
\begin{aligned}
\rho_{y}(\boldsymbol{y}) & =\frac{S(k(\boldsymbol{y})) \rho_{x}\left(\left(\boldsymbol{Y}^{-1}\right)_{x}(\boldsymbol{y})\right)}{\|\Lambda(\boldsymbol{y})\|} \\
& =\frac{S(k(\boldsymbol{y}))}{\|\Lambda\|} \\
\rho_{y} & =\frac{S(|\boldsymbol{n}|)}{\|\Lambda\|} .
\end{aligned}
$$


To get $\|\Lambda\|$, it is easiest to do the following:

$$
\begin{aligned}
& \|\Lambda\|=\sqrt{\left\|\Lambda^{2}\right\|} \\
& =\sqrt{\mid \frac{|\boldsymbol{n}|^{2}}{r^{2}} P_{\perp}+\frac{\boldsymbol{n} \boldsymbol{n}^{T}}{|\boldsymbol{n}|^{2}}} \begin{array}{|}
\mid \\
1 \mid
\end{array}
\end{aligned}
$$

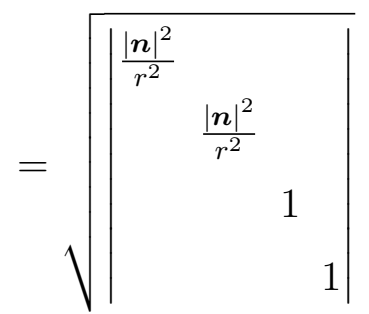

$$
\begin{aligned}
& \|\Lambda\|=\frac{|\boldsymbol{n}|^{2}}{r^{2}} .
\end{aligned}
$$

Substituting this into Eq. (33), we get

$$
\rho_{y}=\frac{r^{2}}{|\boldsymbol{n}|^{2}} S(|\boldsymbol{n}|) .
$$

Now, we can see what choice of $S$ will be most convenient. If $S(k)=k^{2}$ is chosen, $|\boldsymbol{n}|$ is eliminated from the above expression, making the $\boldsymbol{y}$ volume element factor

$$
\rho_{y}=r^{2}
$$

Finally, we get a KEO in $\boldsymbol{y}$ coordinates. From equation 14,

$$
\begin{aligned}
T_{y} & =-\frac{1}{2 m \rho_{y}} \frac{\partial}{\partial y^{a}}\left(\rho_{y} \tau_{y}^{a b} \frac{\partial}{\partial y^{b}}\right) \\
& =-\frac{1}{2 m r^{2}}\left[\begin{array}{ll}
\nabla_{n}^{T} & \partial_{r}
\end{array}\right]\left(r^{2}\left[\begin{array}{ll}
\frac{1}{r^{2}}\left(|\boldsymbol{n}|^{2}-\boldsymbol{n} \boldsymbol{n}^{T}\right) \\
1
\end{array}\right]\left[\begin{array}{l}
\nabla_{n} \\
\partial_{r}
\end{array}\right]\right)
\end{aligned}
$$

Therefore the KEO is

$$
T_{y}=-\frac{1}{2 m r^{2}}\left(\nabla_{n}^{T}\left(\left(|\boldsymbol{n}|^{2}-\boldsymbol{n n}^{T}\right) \nabla_{n}\right)+\partial_{r}\left(r^{2} \partial_{r}\right)\right)
$$

This is a KEO that depends on four coordinates and their derivatives with which one can solve the 3D problem of the motion of a particle in a spherically symmetric potential.

It isn't too difficult to prove (treat $L^{2}$ like a KEO and apply the same procedure) that the first term on the RHS of the previous equation is $L^{2}$, where $L$ is the angular momentum 
operator, so that

$$
\begin{aligned}
T_{y} & =\frac{1}{2 m r^{2}}\left(L^{2}-\partial_{r}\left(r^{2} \partial_{r}\right)\right), \text { where } \\
L^{2} & =-\nabla_{n}\left(\left(|\boldsymbol{n}|^{2} \mathbb{1}-\boldsymbol{n} \boldsymbol{n}^{T}\right) \nabla_{n}\right)
\end{aligned}
$$

This example of this section, although it is simple, illustrates the main ideas. It is possible using Eq. (39) as the KEO to solve a 4D Schroedinger equation to calculate properties of a particle in a spherically symmetric potential. The 4D KEO shows that it is possible to derive a KEO that perserves symmetry with respect to permutation of the $n_{x}, n_{y}, n_{z}$ coordinates. This is not the case when the KEO is written in terms of polar coordinates.

\section{EXAMPLE: A KEO FOR A SYSTEM WITH $N_{a}$ PARTICLES AND $3 N_{a}+3$ COORDINATES}

In principle, it is possible to use $3 N_{a}$ lab-fixed Cartesian coordinates to solve the Schroedinger equation for a system with $N_{a}$ particles. In practice, it is common to use 3 Cartesian coordinates for the centre of mass, three coordinates that specify the orientation of the system, and $3 N_{a}-6$ coordinates that determine its shape. [13, 15, 16] In this section we show that it is possible to write the KEO in $3 N_{a}+3$ coordinates. This might be useful for exploiting symmetry, like in cases where there are $N_{a}$ identical nuclei and it would be useful to preserve all permutation symmetry.

The system has $N_{a}$ particles, labelled by the index $a$, having mass $m_{a}$. The KEO in terms of the Cartesian components of $N_{a}$ lab-fixed position vectors, $\boldsymbol{r}_{\boldsymbol{a}}$, is simple,

$$
T=-\frac{1}{2} \sum_{a=1}^{N_{a}} \frac{\nabla_{a}^{2}}{m_{a}}
$$

In the notation of section II, the regular coordinates are $\boldsymbol{x}=\left\{\boldsymbol{r}_{\boldsymbol{a}}\right\}$. The $3 N_{a}+3$ redundant

coordinates we use are: the three Cartesian components of the vector $\boldsymbol{R}$ from the origin of lab axes to the centre of mass and the three Cartesian components of $N_{a}$ vectors, $\boldsymbol{s}_{\boldsymbol{a}}$, from the centre of mass to each of the particles. In the notation of section II these are the $\boldsymbol{y}$ coordinates: $\boldsymbol{y}=\left(\boldsymbol{R},\left\{\boldsymbol{s}_{\boldsymbol{a}}\right\}\right)$. The function that transforms values of $\boldsymbol{x}$ to values of $\boldsymbol{y}$ is,

$$
\begin{aligned}
\boldsymbol{R} & =\boldsymbol{R}^{\mathbf{0}}(x)=\frac{1}{M} \sum_{a} m_{a} \boldsymbol{r}_{\boldsymbol{a}} \\
\boldsymbol{s}_{\boldsymbol{a}} & =\boldsymbol{s}_{\boldsymbol{a}}^{\mathbf{0}}(x)=\boldsymbol{r}_{\boldsymbol{a}}-\frac{1}{M} \sum_{a} m_{a} \boldsymbol{r}_{\boldsymbol{a}},
\end{aligned}
$$


where $M$ is the total mass. These new coordinates are redundant with the constraint $\sum_{a} m_{a} \boldsymbol{s}_{\boldsymbol{a}}=0$. We obtain a set of augmented coordinates, $\boldsymbol{z}$, by adding to the $\boldsymbol{x}$ coordinates $\boldsymbol{k}=\frac{1}{M} \sum_{a} m_{a} \boldsymbol{s}_{\boldsymbol{a}}$. Since it is the centre of mass of $\left\{\boldsymbol{s}_{\boldsymbol{a}}\right\}, \boldsymbol{k}$ will be labelled $\boldsymbol{S}$ :

$$
\boldsymbol{S}=\frac{1}{M} \sum_{a} m_{a} \boldsymbol{s}_{\boldsymbol{a}}
$$

This makes the augmented coordinates $\boldsymbol{z}=\left(\left\{\boldsymbol{r}_{\boldsymbol{a}}\right\}, \boldsymbol{S}\right)$. A convenient definition of $\boldsymbol{Y}$ (recall that when $\boldsymbol{z}$ is on-shell, i.e. $\boldsymbol{S}=0, \boldsymbol{Y}$ must be consistent with $\boldsymbol{Y}_{\mathbf{0}}$ ) is

$$
\begin{aligned}
\boldsymbol{R} & =\boldsymbol{Y}_{\boldsymbol{R}}(\boldsymbol{x})=\frac{1}{M} \sum_{a} m_{a} \boldsymbol{r}_{\boldsymbol{a}} \\
\boldsymbol{s}_{\boldsymbol{a}} & =\boldsymbol{Y}_{\boldsymbol{s} \boldsymbol{a}}(\boldsymbol{x})=\boldsymbol{S}+\boldsymbol{r}_{\boldsymbol{a}}-\frac{1}{M} \sum_{a} m_{a} \boldsymbol{r}_{\boldsymbol{a}}
\end{aligned}
$$

We now wish to derive the KEO in the $\boldsymbol{z}$ coordinates. This is done by starting with the classical kinetic energy

$$
\boldsymbol{p}_{\boldsymbol{z}}{ }^{T} \boldsymbol{\tau}_{\boldsymbol{z}} \boldsymbol{p}_{\boldsymbol{z}}=\sum_{a} \frac{1}{m_{a}}\left|\boldsymbol{p}_{\boldsymbol{r a}}\right|^{2}
$$

and using Eq. (22), which for this problem is

$$
\sum_{a} \boldsymbol{p}_{\boldsymbol{r a}}{ }^{T} \dot{\boldsymbol{r}}_{\boldsymbol{a}}+\boldsymbol{p}_{\boldsymbol{S}}^{T} \dot{\boldsymbol{S}}=\boldsymbol{p}_{\boldsymbol{R}}^{T} \dot{\boldsymbol{R}}+\sum_{a} \boldsymbol{p}_{\boldsymbol{s a}}{ }^{T} \dot{\boldsymbol{s}}_{\boldsymbol{a}}
$$

We now need $\dot{\boldsymbol{y}}$ in terms of $\dot{\boldsymbol{z}}$. It is

$$
\begin{aligned}
\dot{\boldsymbol{R}} & =\frac{1}{M} \sum_{a} m_{a} \dot{\boldsymbol{r}}_{\boldsymbol{a}} \\
\dot{\boldsymbol{s}}_{\boldsymbol{a}} & =\dot{\boldsymbol{S}}+\dot{\boldsymbol{r}}_{\boldsymbol{a}}-\frac{1}{M} \sum_{a} m_{a} \dot{\boldsymbol{r}}_{\boldsymbol{a}} .
\end{aligned}
$$

Substituting from Eq. (50) and Eq. (51), Eq. (49) becomes,

$$
\begin{aligned}
\sum_{a} \dot{\boldsymbol{r}}_{\boldsymbol{a}}^{T} \boldsymbol{p}_{\boldsymbol{r a}}+\dot{\boldsymbol{S}}^{T} \boldsymbol{p}_{\boldsymbol{S}}= & \sum_{a} \dot{\boldsymbol{r}}_{\boldsymbol{a}}^{T} \boldsymbol{p}_{\boldsymbol{s a}}+\frac{1}{M} \sum_{a} m_{a} \dot{\boldsymbol{r}}_{\boldsymbol{a}}^{T} \boldsymbol{p}_{\boldsymbol{R}} \\
& +\left(\dot{\boldsymbol{S}}^{T}-\frac{1}{M} \sum_{a} m_{a} \dot{\boldsymbol{r}}_{\boldsymbol{a}}^{T}\right) \sum_{a} \boldsymbol{p}_{\boldsymbol{s} \boldsymbol{a}} .
\end{aligned}
$$

From this equation we find, by setting all $\dot{\boldsymbol{z}}=0$ except for $\dot{\boldsymbol{r}}_{\boldsymbol{a}}$,

$$
\begin{aligned}
\dot{\boldsymbol{r}}_{a}^{T} \boldsymbol{p}_{\boldsymbol{r a}} & =\dot{\boldsymbol{r}}_{a}^{T}\left(\boldsymbol{p}_{\boldsymbol{s a}}+\frac{m_{a}}{M}\left(\boldsymbol{p}_{\boldsymbol{R}}-\sum_{b} \boldsymbol{p}_{\boldsymbol{s} b}\right)\right) \\
\boldsymbol{p}_{\boldsymbol{r} \boldsymbol{a}} & =\boldsymbol{p}_{\boldsymbol{s} \boldsymbol{}}+\frac{m_{a}}{M}\left(\boldsymbol{p}_{\boldsymbol{R}}-\sum_{b} \boldsymbol{p}_{\boldsymbol{s} b}\right) .
\end{aligned}
$$


Substituting into equation 48 and simplifying, we get,

$$
\boldsymbol{p}_{\boldsymbol{y}}{ }^{T} \boldsymbol{\tau}_{\boldsymbol{y}} \boldsymbol{p}_{\boldsymbol{y}}=\frac{1}{M}\left|\boldsymbol{p}_{\boldsymbol{R}}\right|^{2}+\sum_{a} \frac{1}{m_{a}}\left|\boldsymbol{p}_{\boldsymbol{s a}}\right|^{2}-\frac{1}{M}\left|\sum_{a} \boldsymbol{p}_{\boldsymbol{s a}}\right|^{2} .
$$

The volume element in this example is trivial since the $\boldsymbol{x}$ volume element and $\Lambda$ are both constant.

\section{NUMERICAL EXAMPLE: THE WATER MOLECULE}

In section $\mathrm{V}$, we demonstrate that, using the formalism we propose, it is possible to calculate exact energy levels of a particle in a spherically symmetric potential using four rather than three coordinates. In section VI, we demonstrate that it is possible to derive a general KEO in $3 N_{a}+3$ coordinates that describes the translation, vibration, and rotation of a polyatomic molecule. In this section, we use a basis set and the formalism of sections II, III, and IV to compute vibrational energy levels of $\mathrm{H}_{2} \mathrm{O}$. This further confirms that the KEO in redundant coordinates is correct.

First, we derive a ro-vibrational KEO in redundant $\boldsymbol{y}$ coordinates, and then we obtain from it the corresponding vibrational KEO. In the notation of section II, the $\boldsymbol{z}$ coordinates are: the three components of $\boldsymbol{s}_{\mathbf{1}}$, the vector for $\mathrm{H}_{1}$ in a frame parallel to the lab frame and with its origin at the centre of mass, the three components of $\boldsymbol{s}_{\mathbf{2}}$, for $\mathrm{H}_{2}$ in the same frame, and an additional coordinate $k$, making seven coordinates in total. (The position of the oxygen nucleus is determined from the condition that the centre of mass is at the origin.) The $\boldsymbol{y}$ coordinates are: two components of $\boldsymbol{\xi}_{\mathbf{1}}$, the molecule-fixed vector for $\mathrm{H}_{1}$, two components of $\boldsymbol{\xi}_{\mathbf{2}}$, the molecule-fixed vector for $\mathrm{H}_{2}$, and three Euler angles. The molecule is restricted to the XY plane in the molecule-fixed frame. There are four vibrational coordinates and only three vibrational degrees of freedom. $k\left(\boldsymbol{\xi}_{\mathbf{1}}, \boldsymbol{\xi}_{\mathbf{2}}\right)$ is chosen so that if $k\left(\boldsymbol{\xi}_{\mathbf{1}}, \boldsymbol{\xi}_{\mathbf{2}}\right)=0$ the Cartesian axes are an Eckart frame. In an Eckart frame, the Coriolis coupling is small close to the equilibrium geometry. [17] Mathematically,

$$
\begin{aligned}
& \boldsymbol{k}\left(\boldsymbol{\xi}_{\mathbf{1}}, \boldsymbol{\xi}_{\mathbf{2}}\right)=m_{p} \boldsymbol{\xi}_{\mathbf{1}}^{e \boldsymbol{q}} \times \boldsymbol{\xi}_{\mathbf{1}}+m_{p} \boldsymbol{\xi}_{\mathbf{2}}^{e \boldsymbol{q}} \times \boldsymbol{\xi}_{\mathbf{2}}+m_{O} \boldsymbol{\xi}_{\boldsymbol{O}}^{e \boldsymbol{q}} \times \boldsymbol{\xi}_{O} \\
& \boldsymbol{k}\left(\boldsymbol{\xi}_{\mathbf{1}}, \boldsymbol{\xi}_{\mathbf{2}}\right)=0 \text { (on-shell) }
\end{aligned}
$$

where $\boldsymbol{\xi}_{\boldsymbol{i}}$ is a $2 \mathrm{D}$ vector in the molecule-fixed frame and $\boldsymbol{\xi}_{\boldsymbol{i}}{ }^{e q}$ is the equilibrium vector. Just as in the $\boldsymbol{z}$ coordinates, $\boldsymbol{\xi}_{\boldsymbol{O}}$ is determined from $\boldsymbol{\xi}_{\mathbf{1}}$ and $\boldsymbol{\xi}_{\mathbf{2}} \cdot m_{p}$ is the mass of a proton and $m_{O}$ 
is the mass of the oxygen nucleus. Two of the three constraints of Eq. (54) are automatically satisfied because the molecule is planar. [18] The remaining non-trivial constraint defines the shell. Both the $\boldsymbol{\tau}_{\boldsymbol{y}}$ matrix and $\rho_{y}$ depend on the coordinates. Since the variation in each position is relatively small compared to the magnitude of the equilibrium position, the approximation $\boldsymbol{\xi}_{\boldsymbol{a}} \approx \boldsymbol{\xi}_{\boldsymbol{a}}^{\boldsymbol{e q}}$ was made in the $\boldsymbol{\tau}_{\boldsymbol{y}}$ and $\rho_{y}$. Despite these approximations, the energy levels we compute are very close to the experimental levels. They therefore do not impede our ability to validate our approach for computing in redundant coordinates. To obtain the $J=0 \mathrm{KEO}$ we simply discard all terms with derivatives with respect to the Euler angles. This leads to the following Hamiltonian:

$$
\begin{aligned}
H & =\frac{1}{2 m_{p}}\left|\boldsymbol{p}_{\mathbf{1}}\right|^{2}+\frac{1}{2 m_{p}}\left|\boldsymbol{p}_{\mathbf{2}}\right|^{2} \\
& -\frac{1}{2\left(m_{O}+2 m_{p}\right)}\left|\boldsymbol{p}_{\mathbf{1}}+\boldsymbol{p}_{\mathbf{2}}\right|^{2}-\frac{1}{2 I^{e q}} L_{z}^{2}+V, \text { where } \\
L_{z} & =\boldsymbol{\xi}_{\mathbf{1}}^{\boldsymbol{e q}} \times \boldsymbol{p}_{\mathbf{1}}+\boldsymbol{\xi}_{\mathbf{2}}^{e \boldsymbol{q}} \times \boldsymbol{p}_{\mathbf{2}}, \\
\boldsymbol{p}_{\boldsymbol{a}} & =-i\left[\frac{\partial}{\partial \xi_{a}^{x}} \frac{\partial}{\partial \xi_{a}^{y}}\right]^{T}, \text { and } \\
I^{e q} & =\sum_{a} m_{a}\left|\boldsymbol{\xi}_{\boldsymbol{a}}^{e \boldsymbol{q}}\right|^{2} .
\end{aligned}
$$

The potential used was that of Polyansky, Jensen, and Tennyson[19].

The basis is a pruned direct product sinc discrete variable representation (DVR) basis. [20] To make the direct product basis, we use $N$ sinc DVR functions for each coordinate. The size of the product basis is $N^{4}$. We reduce the size of the basis by retaining only functions centred about points within $\varepsilon$ of the physical region (shell), which is defined by $k\left(x_{1}, y_{1}, x_{2}, y_{2}\right)=0$. This can be done by using the inner product

$$
\langle\psi, \phi\rangle=\int d x_{1} d x_{2} d y_{1} d y_{2} W\left(k\left(x_{1}, x_{2}, y_{1}, y_{2}\right) / \varepsilon\right) \psi\left(x_{1}, x_{2}, y_{1}, y_{2}\right)^{*} \phi\left(x_{1}, x_{2}, y_{1}, y_{2}\right)
$$

where $W(u)$ is the unit square window, equal to 1 when $u$ is within 1 of the origin and zero otherwise.

We have made no attempt to refine the computational method to make it useful for computing many energy levels. Our goal is to compute a few levels to demonstrate that the redundant formalism of sections II, III, and IV is correct. Computed energy levels are degenerate, although the actual levels are not. This is because we are effectively solving 


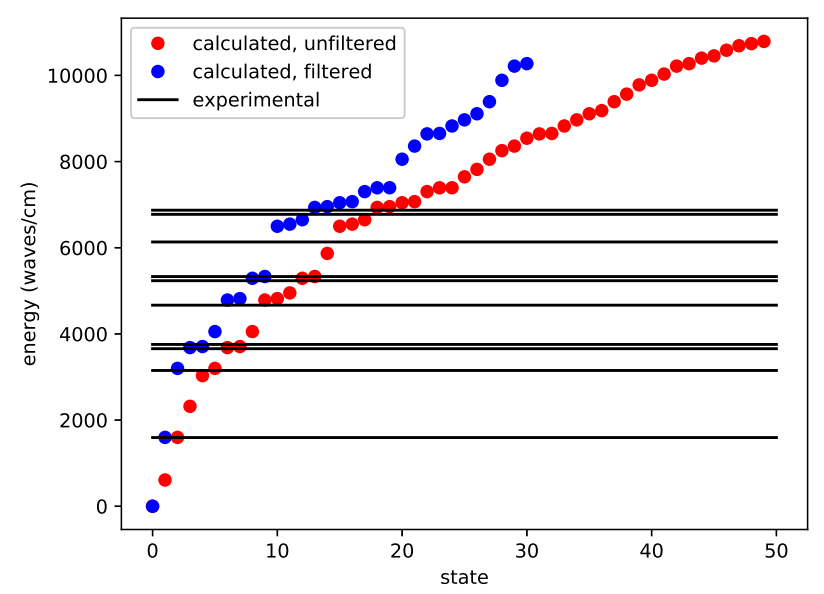

FIG. 1: Vibrational band origins of $\mathrm{H}_{2} \mathrm{O}$

a 4D time-independent Schroedinger equation in which the fourth coordinate is uncoupled to the three physical coordinates. Thus, the higher the $\varepsilon$ the greater the degeneracy of the energy levels. The method also produced spurious eigenvalues. Their values were sensitive to the choice of $\varepsilon$ and it was therefore simple to filter out the spurious eigenvalues by solving the problem twice with slightly different values of $\varepsilon$ and keeping the energies obtained with both $\varepsilon$. The lowest levels we compute are shown in figure 1. The experimental energy levels used for comparison were obtained from Ref. [21]. We have pushed the calculation only far enough to demonstrate that the new ideas are working for this example. Relative errors for the four excited states are : 0.00236134, 0.01524822, 0.00697031, -0.0131596. At higher energies the calculation is less well converged and there are more spurious eigenvalues.

\section{CONCLUSION}

The inner product of section III and the KEO of section IV make it possible to solve the Schroedinger equation in redundant coordinates. We have presented several examples to demonstrate that the ideas work. Standard methods for deriving KEOs are all justified by the chain rule. It works well (although the resulting operators are often complicated) when one wishes to derive a KEO depending on $M$ coordinates from another KEO that depends on a different set of $M$ coordinates. When one wishes to use $N$ redundant coordinates $(N>M)$, the chain rule by itself is not sufficient. If the $N$ dimensional basis functions have 
amplitude in non-physical regions then to obtain exact solutions (i.e. those one would obtain by using a KEO in $M$ coordinates), then it is necessary to use a KEO that incorporates information about the constraint. In this paper we derive such a KEO. To the best of our knowledge, this has never been done. There are known recipes for deriving a KEO in a subspace of configuration space obtained by fixing some of the $M$ coordinates. Rather than decreasing the number of coordinates to facilitate obtaining (approximate) solutions, we increase the number of coordinates, but derive equations that make it possible to compute exact solutions. Both the (analytic) 4D KEO for a single particle in 3D and the (numerical) $4 \mathrm{D} \mathrm{KEO}$ for the vibration of $\mathrm{H}_{2} \mathrm{O}$ demonstrate that the ideas work.

Several improvements of the computational procedure are possible. To start with, using basis functions with amplitude in the non-physical region increases the size of the required basis. Of course, one wishes to minimize the size of the basis, so it would be best to choose basis functions in redundant coordinates that have little (or no) amplitude in the non-physical region. Approximating the shell with an $M$-simplex mesh and applying a finite element method accomplishes this. There are two obvious potential advantages to using redundant coordinates. 1) It is known that molecular potentials can be represented using only a small number of terms each of which depends on a small subset of redundant coordinates, but to exploit this advantage one needs to be able to solve the Schroedinger equation in the coordinates on which the potential depends. 2) Often applying symmetry operators to non-redundant coordinates transforms them in a complicated way. The action of permutation symmetry operators on inter-atomic distances - redundant for molecules with more than four atoms - is very simple. There are many other possible applications of the

redundant formalism we propose. For example, to use (the four) quaternion components as coordinates for rotation (three degrees of freedom) one would need to know how to deal with redundant coordinates.

\section{Acknowledgments}

This work has been supported by the Natural Sciences and Engineering Research Council of Canada. 
[1] Chen Qu, Qi Yu, and Joel M. Bowman, Annual Review of Physical Chemistry, 69, 151-175 (2018)

[2] Tucker Carrington, The Journal of Chemical Physics 146, 120902 (2017)

[3] J. Bowman, T. Carrington and H.-D. Meyer, Mol. Phys. 106, 2145-2182 (2008)

[4] Jiayan Wu, Xinchuan Huang, Stuart Carter, Joel M. Bowman, Chemical Physics Letters 426 $285289(2006)$

[5] X.-G Wang and T. Carrington, Journal of Physical Chemistry, 105, 2575-2581 (2001)

[6] Gustavo Avila and Tucker Carrington, Journal of Chemical Physics 131174103 (2009)

[7] L. Kaplan, N. T. Maitra, and E. J. Heller, Phys. Rev. A 56, 2592 (1997).

[8] A. M. Dirac, Can. J. Math 2, 129 (1950).

[9] B. DeWitt, Rev. Mod. Phys. 29, 377 (1957).

[10] Fabien Gatti and Christophe Iung, Physics Reports, 484, 1-69 (2009)

[11] Xiao-Gang Wang and Tucker Carrington Jr, J. Chem. Phys. 115, 9781-9796 (2001)

[12] C Fabri, E Matyus, AG Csaszar, The Journal of chemical physics 134, 074105 (2011)

[13] N. C. Handy, MOLECULAR PHYSICS, 61, 207-223 (1987),

[14] Philip R. Bunker and Per Jensen, Molecular Symmetry and Spectroscopy, 2nd Ed., NRC Research Press (2006)

[15] D. Papousek and M. R. Aliev, Molecular Vibrational and Rotational Spectra, Elsevier Scientific Publishing Company, New York 1982.

[16] Robert G. Littlejohn and Matthias Reinsch Rev. Mod. Phys. 69, 213 (1997)

[17] James D. Louck and Harold W. Galbraith, Reviews of Modern Physics, 48, 69 (1976)

[18] H Wei and T Carrington Jr Chemical Physics Letters, 287, 289 (1998)

[19] O. L. Polansky, P. J. Jensen, and J. Tennyson, J. Chem. Phys. 105, 6490 (1996)

[20] Daniel T. Colbert and William H. Miller, J. Chem. Phys. 96, 1982 (1992)

[21] S. V. Shirin, O. L. Polyansky, N. F. Zobov, A. G. Császár, and J. Tennyson, J. Mol. Spectrosc. 236, $216(2006)$ 